\title{
КОМОРБИДНЫЕ РАССТРОЙСТВА У ДЕТЕЙ С СИНДРОМОМ ДЕФИЦИТА ВНИМАНИЯ С ГИПЕРАКТИВНОСТЬЮ
}

\section{Саидходжаева С.Н.*}

Ташкентский педиатрический медицинский институт, Ташкент, Узбекистан

В статье на основании обследования 86 детей рассматривается проблема коморбидных расстройств при синдроме дефицита внимания и гиперактивности, которые встречаются у 69,8\% больных и приводят к дополнительным сложностям социальной, школьной и внутрисемейной адаптации. Структура коморбидных расстройств характеризуется наличием оппозиционно-вызывающих расстройств поведения (43,0\%) и различными формами тревожных расстройств (39,5\%). У 9,3\% детей с данным заболеванием встречались тики, у 5\% - логоневроз, ночной энурез - у 17,4\%.

Ключевые слова: синдром дефицита внимания и гиперактивности, коморбидность, дети, диагностика.

Актуальность исследования синдрома дефицита внимания с гиперактивностью (СДВГ) определяется высокой частотой данного расстройства в детской популяции. СДВГ широко представлен в детской популяции. Его распространенность колеблется от 2 до $12 \%$ (в среднем 3-7\%), чаще встречается у мальчиков, чем у девочек (в среднем соотношение - 3:1) [1, 2]. СДВГ - это название этиологически гетерогенной группы нарушений поведения у детей старше 5-летнего возраста. Эти изменения поведения сопровождаются нарушением внимания и гиперактивностью, потенциально приводящими к неуспеваемости (нарушения обучения), антисоциальному поведению, снижению качества жизни [1-5].

СДВГ может встречаться как в изолированном виде, так и в сочетании с другими эмоциональными и поведенческими нарушениями, оказывая негативное влияние на обучение и социальную адаптацию.

Дополнительные сложности внутрисемейной, школьной и социальной адаптации у детей с СДВГ могут быть связаны с формированием сопутствующих нарушений, которые развиваются на фоне СДВГ как основного заболевания не менее чем у $70 \%$ пациентов [1, 2]. Наличие коморбидных расстройств может приводить к утяжелению клинических проявлений СДВГ, ухудшению отдаленного прогноза и снижению эффективности основной терапии по поводу СДВГ. Сопутствующие СДВГ нарушения поведения и эмоциональные расстройства рассматриваются в качестве неблагоприятных прогностических факторов для длительного, вплоть до хроническо*e-mail: doctor-saida1986@mail.ru го, течения СДВГ [4].

Коморбидные расстройства при СДВГ представлены следующими группами: экстернализированные (оппозиционно-вызывающее расстройство, расстройство поведения), интернализированные (тревожные расстройства, расстройства настроения), когнитивные (нарушения развития речи, специфические трудности обучения - дислексия, дисграфия, дискалькулия), двигательные (статико-локомоторная недостаточность, диспраксия развития, тики). Другими сопутствующими СДВГ расстройствами могут быть нарушения сна (парасомнии), энурез, энкопрез $[8,9]$.

Таким образом, проблемы в обучении, поведении и эмоциональной сфере могут быть связаны как с непосредственным влиянием СДВГ, так и с коморбидными расстройствами, которые должны быть своевременно диагностированы и рассматриваться в качестве показаний для дополнительного назначения соответствующего лечения.

Целью данной работы является изучения характерных особенностей коморбидных состояний у детей с СДВГ.

Материалы и методы исследования в основу исследования положены данные анамнестического и клинико-неврологического обследования 86 детей с СДВГ в возрасте 5-12 лет

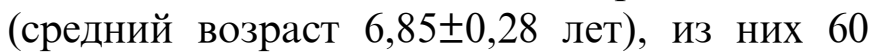
мальчиков $(69,8 \%)$ и 26 девочек $(30,2 \%)$ $(\mathrm{P}<0,05)$.

Всем обследованным детям было проведён углубленный анамнестический анализ, полученных из амбулаторных анкет, клинико-нев- 
рологическое обследование, данные диагностического классификатора СДВГ - Американской психиатрической ассоциации DSM-IV.

Результаты исследования. Анализируемые факторы риска формирования СДВГ были разделены на две группы: медико-биологические, и психосоциальные. Среди медикобиологических факторов у $37,2 \%$ детей с СДВГ был отмечен отягощенный акушерский анамнез. Гестоз выявлен у 22,1\%, хроническая фетоплацентарная недостаточность на фоне гестоза у 15,1\%, угрозу прерывания беременности у $27,9 \%$. Экстрагенитальные заболевания в период беременности у 55,8\%.

Из неблагоприятных факторов интранатального периода была выявлена высокая частота патологии родов $(57,0 \%)$. Наиболее частыми причинами патологии родов были: нарушения длительности родов $(22,1 \%)$, слабость родовой деятельности и использование родостимуляции $(27,9 \%)$. Как следствие неблагоприятного течения антенатального и интранального периодов развитие перинатального поражения ЦНС отмечалось у $65,1 \%$ детей. Отягощенную по СДВГ наследственность выявляли у 37,2\% детей. Опрос 58 матерей детей с СДВГ выявил высокий уровень психоэмоциональных стрессов, перенесенных в отдельные периоды беременности и на протяжении всей беременности $(78,6 \%)$, депрессивное состояние в течение всего периода беременности $(17,8 \%)$, "страх родов" $(32,1 \%)$.

Среди социально-бытовых наиболее значимыми были: неудовлетворительные условия проживания - у 37,2\% семей, недостаточное обеспечение детей собственным рабочим местом (детским уголком - 52,3\%, письменным столом - 69,8\%) и особенно, спортивными тренажерами $(20,9 \%)$.

Неблагоприятными семейными психологическими факторами являлись: конфликты между родителями по вопросам воспитания $(25 \%)$, неспокойная обстановка в семье $(21,4 \%)$, частые ссоры между родителями в присутствии ребенка $(17,8 \%)$, алкоголизм отца $(14,3 \%)$, воспитание в неполной семье $(10,7 \%)$. Нарушение режима дня выявлено в $46,4 \%$.

При неврологическом осмотре отмечалась, в основном, негрубая диффузная органическая симптоматика. У 32,6\% детей выявлены дисфункции черепно-мозговых нервов. Зафиксированы негрубые расстройства в двигательной и координаторной сферах: изменения мышечного тонуса - 67,4\%, гиперрефлеския - 78,3\%, элементы статической атаксии $72,1 \%$, элементы динамической атаксии $67,4 \%$, дисдиадохокинез - 70,9\%.

По данным результатов DSM-IV наиболее частыми клиническими вариантами синдрома были: гиперактивность (у 21 детей $24,4 \%$ ), импульсивность (у 26 детей - 30,2\%) и сочетанная форма (гиперактивность + дефицит внимания) - у 22 детей - 25,6\%, несколько реже выявлялся вариант с преимущественным дефицитом внимания (у 17 детей 19,8\%)

Среди диагностированных коморбидных расстройств наиболее распространенными оказались оппозиционно-вызывающее расстройство поведения (43,0\% - 37 детей) и различные формы тревожных расстройств (39,5\% - 34 ребенка). Сочетание нескольких форм тревожных расстройств имело место быть у 9 пациентов с СДВГ (6 мальчика, 3 девочки). Показано, что при с СДВГ в сочетании с тревожными расстройствами наблюдается более выраженная клиническая симптоматика, чем в случаях каждого из заболеваниях по отдельности, а также более значительные нарушения адаптации, социальнопсихологического функционирования и трудности школьного обучения [10,11].

Значительно чаще встречались тики - у 9 детей $(9,3 \%)$, у 15 - ночной энурез $(17,4 \%)$ и у 5детей - логоневроз (5,8\%). Тики были простыми моторными и вокальными, характеризовались длительным течением. Энурез в большинстве случаев имел форму неврозоподобного, вторичного энуреза. Всего коморбидные расстройства выявлены у 60 из 86 обследованных детей с СДВГ, т. е. в 69,8\% случаев. Из них у 32 пациентов $(53,3 \%)$ отмечалось одно коморбидное расстройство, у 18 $(30 \%)$ - два, у $9(15 \%)$ - три и у одного $(1,7 \%)$ четыре коморбидных расстройства. Доля случаев "чистого" СДВГ, не сопровождавшегося данными коморбидными расстройствами, 
составила 30,2\%.

Других расстройств, которые по данным литературы могут сопутствовать СДВГ, в том числе асоциальных расстройств поведения и депрессий, у наших пациентов не было выявлено. Это может обьясняться двумя обстоятельствами: 1) Дети с СДВГ, вошедшие в группу контроля, обследовались в амбулаторных условиях, а не в стационаре. 2) частота встречаемости асоциальных и депрессивных состояний увеличивается к периоду пубертатности, тогда как возраст наших пациентов составил от 5 до 11 лет [7].

Таким образом, сопутствующие СДВГ расстройства представляют серьезную проблему. Они должны быть вовремя диагностированы, поскольку их поздние выявления и несвоевременная терапия часто служат предикторами неблагоприятной возрастной динамики СДВГ в подростковом и молодом взрослом возрасте, нарастания трудностей социальной

\section{ЛИТЕРАТУРА}

1. Баркли РА. Дети с вызывающим поведением. Клиническое руководство по обследованию ребенка и тренингу родителей. Пер. с англ. М.: Теревинф, 2011. - 272 с..

2. Безруких М.М. Дети с СДВГ: причины, диагностика, комплексная помощь. - Москва: Медицина, 2009. $250 \mathrm{c}$.

3. Белопольская Н.Л. Детская патопсихология. - Москва: ГЕОТАР, 2004. - 230 с.

4. Воронин Н.А. Особенности памяти у детей с синдромом дефицита внимания и гиперактивности 6-8 лет // Специальная психология. - 2008. - №2(16). - С. 53-61.

5. Заваденко НН, Суворинова НЮ. Синдром дефицита внимания с гиперактивностью: выбор оптимальной продолжительности лекарственной терапии // Журн. неврол. и психиатр. им. С.С.Корсакова. - 2011. №111(10). - С. 28-32.

6. Эверт Л.С., Полуэктова Т.С. Частота встречаемости и структура клинических вариантов синдрома дефицита внимания с гиперактивностью у школьников Республики Хакасия (на примере г. Абакана) // Медицина и здравоохранение: материалы междунар. науч. конф. адаптации пациентов. Кроме того, случаи СДВГ с коморбидными расстройствами требуют специальных подходов к лечению.

\section{Выводы}

1. Основными проявлениями СДВГ являются гиперактивность и импульсивность, а также дефицит внимания.

2. Факторами риска формирования СДВГ являются перинатальное повреждение ЦНС $(65,1 \%)$,неблагоприятные социально-психологические воздействия $(60,7 \%)$.

3. СДВГ может встречаться как в изолированном виде, так и в сочетании с другими эмоциональными и поведенческими нарушениями, оказывая негативное влияние на обучение и социальную адаптацию.

4. Сопутствующие СДВГ нарушения поведения и эмоциональные расстройства рассматриваются в качестве неблагоприятных прогностических факторов для длительного, вплоть до хронического, течения СДВГ

(г. Чита, ноябрь 2012 г.). - Чита: Издательство Молодой ученый, 2012. - С. 48-51.

7. Заваденко Н.Н., Суворинова Н.Ю. Коморбидные расстройства при синдроме дефицита внимания с гиперактивностью у детей // Журн. неврологии и психиатрии им. С.С. Корсакова. 2007. Т. 107, № 7. С. $39 ? 44$.

8. Balint S., Czobor P., Meszaros A., Simon V., Bitter I. Neuropsychological impairments in adult attention deficit hyperactivity disorder: a literature review. // Psychiatr Hung. - 2008. - Vol. 23 (5). - P. 324-35.

9. Pennington B.F. Diagnosing Learning Disorders. //A Neuropsychological Framework. New York, London. 2009. - P. 355.

10. Tannock R. Attention-deficit/Hyperactivity Disorder with Anxiety Disorders // Attention-deficit Disorders and Comorbidities in Children, Adolescents, and Adults / ed. by T.E. Brown. Washington, 2000. P. $125 ? 17$

11. The Revised Conners' Parent Rating Scale (CPRS-R): Factor Structure, Reliability, and Criterion Validity / C.K. Conners, G. Sitarenios, J.D. Parker, J.N. Epstein // J. Abnorm. Child Psychol. 1998. 26(4). P. 257-268. 


\title{
XÜLASə \\ DIQQЭT DEFISITII Və HIPERAKTIVLIK SINDROMU ILə \\ UŞAQLARDA KOMORBID POZUNTULAR
}

\author{
Saidhocayeva S.N. \\ Daşkənd Pediatrik Tibb İnstitutu, Daşkənt, Özbəkistan
}

Təqdim edilmiş məqalədə 86 uşağın müayinəsi əsasında diqqətin defisiti və hiperaktivlik sindromu zamanı komorbid pozuntular tədqiq edilir ki, bu da $69,8 \%$ xəstədə rast gəlinir və əlavə olaraq sosial, məktəb və ailədaxili adaptasiya probleminə səbəb olur. Komorbid pozuntuların strukturu davranışın açıq-oppozision pozulmalarının $(43,0 \%)$ və həyacan pozulmalarının müxtəlif formalarının $(39,5 \%)$ olması ilə xarakterizə olunur. Bu xəstəliyi olan uşaqların 9,3\%-də tiklərə, 5\%-də loqonevroza, 17,4\%-də gecə enurezinə rast gəlinmişdir. Açar sözlər: diqqət defisiti və hiperaktivlik sindromu, komorbidlik, uşaqlar, diaqnostika.

\section{SUMMARY}

\section{COMORBID DISORDERS OF ATTENTION DEFICIT HYPERACTIVITY DISORDER IN CHILDREN}

\author{
Saidkhodjaeva S.N. \\ Tashkent Pediatric Medical Institute, Tashkent, Uzbekistan
}

In the article on the basis of a survey of 86 children the problem of comorbidity with attention deficit hyperactivity disorder, which are found in $69.8 \%$ of patients and lead to additional complications of intra-family, school and social adaptation. The structure is characterized by the presence of comorbid disorders, oppositional defiant behavior disorders (43.0\%) and various forms of anxiety disorders $(39.5 \%)$. At $9.3 \%$ of children with the disease have met tics, $17.4 \%$ - nocturnal enuresis and 5.8 - faecal incontinence.

Keywords: attention deficit hyperactivity disorder, comorbidity,children, diagnosis.

Redaksiyaya daxil olub: 03.09.2015

Çapa tövsiya olunub: 29.09.2015

Rayçi: t.ü.f.d. Oliyev R.R. 